\title{
Barley ROP-Interactive Partner-a organizes into RAC1- and MICROTUBULE-ASSOCIATED ROP-GTPASE ACTIVATING PROTEIN 1-dependent membrane domains
}

\author{
Caroline Hoefle, Christopher McCollum and Ralph Hückelhoven * (D)
}

\begin{abstract}
Background: Small ROP (also called RAC) GTPases are key factors in polar cell development and in interaction with the environment. ROP-Interactive Partner (RIP) proteins are predicted scaffold or ROP-effector proteins, which function downstream of activated GTP-loaded ROP proteins in establishing membrane heterogeneity and cellular organization. Grass ROP proteins function in cell polarity, resistance and susceptibility to fungal pathogens but grass RIP proteins are little understood.

Results: We found that the barley (Hordeum vulgare L.) RIPa protein can interact with barley ROPs in yeast. Fluorescenttagged RIPa, when co-expressed with the constitutively activated ROP protein CA RAC1, accumulates at the cell periphery or plasma membrane. Additionally, RIPa, locates into membrane domains, which are laterally restricted by microtubules when co-expressed with RAC1 and MICROTUBULE-ASSOCIATED ROP-GTPASE ACTIVATING PROTEIN 1. Both structural integrity of MICROTUBULE-ASSOCIATED ROP-GTPASE ACTIVATING PROTEIN 1 and microtubule stability are key to maintenance of RIPa-labeled membrane domains. In this context, RIPa also accumulates at the interface of barley and invading hyphae of the powdery mildew fungus Blumeria graminis f.sp. hordei.

Conclusions: Data suggest that barley RIPa interacts with barley ROPs and specifies RAC1 activity-associated membrane domains with potential signaling capacity. Lateral diffusion of this RAC1 signaling capacity is spatially restricted and the resulting membrane heterogeneity requires intact microtubules and MICROTUBULE-ASSOCIATED ROP-GTPASE ACTIVATING PROTEIN 1. Focal accumulation of RIPa at sites of fungal attack may indicate locally restricted ROP activity at sites of fungal invasion.
\end{abstract}

Keywords: Arabidopsis thaliana, Hordeum vulgare, Interactor of constitutive active ROPs, Membrane asymmetry, Microtubule, RAC GTPase, ROP GTPase, Susceptibility, Resistance

* Correspondence: hueckelhoven@wzw.tum.de

Chair of Phytopathology, TUM School of Life Sciences Weihenstephan,

Technical University of Munich, Emil Ramann Str. 2, 85354 Freising, Germany

C C The Author(s). 2020 Open Access This article is licensed under a Creative Commons Attribution 4.0 International License, which permits use, sharing, adaptation, distribution and reproduction in any medium or format, as long as you give appropriate credit to the original author(s) and the source, provide a link to the Creative Commons licence, and indicate if changes were made. The images or other third party material in this article are included in the article's Creative Commons licence, unless indicated otherwise in a credit line to the material. If material is not included in the article's Creative Commons licence and your intended use is not permitted by statutory regulation or exceeds the permitted use, you will need to obtain permission directly from the copyright holder. To view a copy of this licence, visit http://creativecommons.org/licenses/by/4.0/ The Creative Commons Public Domain Dedication waiver (http://creativecommons.org/publicdomain/zero/1.0/) applies to the data made available in this article, unless otherwise stated in a credit line to the data. 


\section{Background}

In plants, ROP (RHO of plants) small GTPases are the only members of the RHO protein family, which consists of several subfamilies (RHO, RAC, CDC42, Rnd und RhoBTB) in mammals [1,2]. ROPs organize a bunch of cellular processes as signaling GTPase. Among the most prominent ROP-regulated events are the subcellular organization of the cytoskeleton and vesicular traffic [3]. ROP-regulated cellular organization is crucial for normal plant development e.g. in polar cell growth or asymmetric cell division but also in interaction with the environment e.g. in regulation of stomata aperture or in interaction with pathogens. ROP activity is tightly regulated via proteins that facilitate hydrolysis and exchange of ROP-bound nucleotides. ROP-GDP is the signalinginactive form of ROP and can be further controlled by ROP-GDIs (ROP-guanine nucleotide dissociation inhibitors) that bind to ROP-GDP. ROP-GDIs support cytosolic localization of ROPs most likely by direct binding of isoprenyl-residues at the C-terminus of type I ROPs, which carry a CAAX-box prenylation motif. ROP-GDP further can interact with different types of ROP guanine nucleotide exchange factors (GEFs), which support the release of GDP and binding of GTP. This turns the protein into activated ROP-GTP that signals downstream. ROP GTPase-activating proteins (GAPs) then can switch off activated ROPs again by supporting the otherwise low intrinsic GTPase function of ROPs and facilitating GTP hydrolysis [3, 4]. Negatively charged lipids at the inner leaflet of the plasma membrane may further function in ROP-positioning and signaling $[5,6]$.

In barley, distinct ROP GTPases are susceptibility factors in the interaction with the powdery mildew fungus Blumeria graminis f.sp. hordei (Bgh). Several ROPs, when constitutively activated (CA) by mutations in the GTPase domain, can support invasion of epidermal cells by fungal hyphae, which subsequently form a haustorium as a feeding cell in a living epidermal cell of barley [7]. Vice versa, sequence-specific RNA interference for silencing $R A C B$ renders barley less susceptible to fungal invasion and limits disease development $[8,9]$. RACB's physiological function is described in polar cell development during formation of root hairs and leaf stomata complexes [10]. Since Bgh appears to target RACB directly by an virulence effector, it was suggested that the fungus exploits a plant polar cell developmental pathway for the accommodation of haustoria in living barley cells [11]. Another barley ROP called RAC1, has a less well understood function in the interaction with $B g h$. Transient expression of CA RAC1 in single epidermal cells did not render barley supersusceptible [7]. However, the same open reading frame, when stably expressed in transgenic barley, supported fungal penetration but also the generation of reactive oxygen species in non-penetrated cells. CA RAC1 further supported barley resistance to the rice blast fungus Magnaporthe oryzae, similar to what was reported before for the function of rice $\mathrm{RACl}$, which is $86 \%$ identical to barley RAC1 [4, 12].

The barley genome encodes several predicted ROPGAP proteins, but only the MAGAP1 (MICROTUBULE-ASSOCIATED ROP-GTPASE ACTIVATING PROTEIN 1) has been characterized thus far. MAGAP1 contains a CRIB motif (for CDC42/RAC-Interactive Binding) and can bind to both RACB and RAC1 and is associated with microtubules. However, besides a localization at MTs, MAGAP1 positions at the cell periphery when recruited by CA RACB and to a minor extent in the cytoplasm. MAGAP1 is considered as a functional antagonist of RACB because MAGAP1 overexpression limits susceptibility whereas MAGAP1 silencing supports susceptibility to penetration by $B g h$ [9]. Additionally, potentially ROP-regulated stability and polarity of MTs is associated with resistance to fungal penetration in barley $[9,11,13]$.

ROP-GTP signals downstream via protein-protein interaction that depends of the ROP-loaded nucleotide and hence the three-dimensional constitution of ROPs. Proteins, which mediate ROP downstream effects, are commonly called ROP-effectors. However, not all ROPeffectors directly fulfill a function in cellular organization but instead are suggested to be scaffolds or adapter proteins that link activated ROPs with downstream factors. RIPs (ROP-Interactive Partner; the founding members of that ROP effector family were first also called Interactor of Constitutive Active ROPs [ICR] [14]) and RICs (ROPInteractive CRIB motif-containing proteins) are such ROP-effectors without known biochemical but potential ROP-scaffolding function [3, 14-16].

ICR/RIP proteins were described in some detail for the dicot model Arabidopsis thaliana. ICR/RIPs possess an overall little conserved amino acid sequence, when compared between protein family members. However, they show a conserved QEEL/QDEL motif of unknown function in the $\mathrm{N}$-terminal part of the proteins and a conserved QWRKAA motif, which is involved in binding ROPs. ICR/RIPs have been described to bridge between activated ROP proteins and downstream interaction partners that act in plant membrane and cell polarity by interaction with components of microtubule (MT) organization or vesicle trafficking $[14,15,17-20]$. For monocot ICR/RIP proteins, however, little information is available.

Here we show that the barley ICR/RIP protein RIPa interacts with barley ROPs and specifies RAC1 activityassociated membrane domains. Thereby, RIPa together RAC1 and MAGAP1 build a ROP signaling module, which creates asymmetric plasma membrane domains. 
Lateral diffusion of RAC1 activity, which is indicated by recruitment of RIPa into membrane domains, is spatially restricted by MTs and the resulting membrane heterogeneity requires intact MTs and MAGAP1.

\section{Results}

\section{Barley RIPa is a ROP binding protein}

Because ROP signaling and microtubule organization seems to be important in interaction of barley and $B g h$, we looked for candidate proteins that potentially are involved in both processes. Arabidopsis thaliana RIP3 (also called ICR5 and microtubule depletion domain 1, MIDD1) can interact with ROPs and MT-associated kinesin13A in planta [17]. Oda and co-workers found RIP3/MIDD1 to be part of a ROP regulatory module, which determines MT organization and subcellular cell wall deposition in xylem cells $[18,19,21]$. We therefore speculated that barley proteins with homology to RIP3 (AT3G53350) can act in ROP signaling during fungal invasion or defensive plant cell wall apposition (see also [22]). The barley locus HORVU3Hr1G087430.11 (protein accession F2DI37_HORVV) encodes the barley protein with similarity to Arabidopsis RIP3 and RIP2 and contains the ICR/RIP-characteristic QDEL and QWRKAA motifs in the $\mathrm{N}$-terminal or C-terminal protein domains, respectively. However, the rest of the protein is little conserved in Arabidopsis, protein identity between these Arabidopsis and barley RIP proteins is $36 \%$, and the barley protein is with 510 amino acids much longer than Arabidopsis RIP3 with 396 amino acids [22]. We thus named the barley protein RIPa instead of RIP3 because we cannot predict whether barley $\mathrm{RIPa}$ is indeed the orthologue of Arabidopsis RIP3. To confirm that RIPa might be a ROP-binding protein, we checked protein-protein interaction in a targeted yeast-twohybrid assay and found that RIPa interacts with RACB and RAC1 from barley as well as with CA versions of these proteins but not with dominant negative versions (Fig. 1). RIPa appears thus to be able to interact in yeast with so-called type I ROPs carrying a carboxyterminal CAAX-box prenylation signal (RACB) as well as with type II ROPs that are predicted to be constitutively palmitoylated (RAC1) [7, 23].

\section{ROPs can influence subcellular localization of RIPa}

We then studied subcellular localization of RIPa by confocal laser scanning microscopy. When we expressed a yellow fluorescing fusion protein, YFP-RIPa, the fluorescence signal was always detectable in the cytoplasm and strong in undefined speckles, which were little mobile and only co-localized partially with the MT-marker RFP-MAGAP1-Cterm, which contains the MT-binding domain of MAGAP1 but does not interact with ROPs because it lacks the ROP-binding CRIB and GAP domains [9] (see below and (Fig. 2).

We hypothesized that the speckled localization of YFP-RIPa represents protein aggregates that form when a scaffold protein is expressed without a corresponding amount of protein binding partners. RIPa could also interact with itself in yeast-2-hybid assays and hence might form multimers when ectopically expressed (Additional file 1, Figure S1). To test, whether co-expression of potential binding partners might change subcellular localization of YFP-RIPa, we co-expressed RAC1, CA RAC1 and DN RAC1. Astonishingly, both expression of RAC1 or CA RAC1 completely changed subcellular
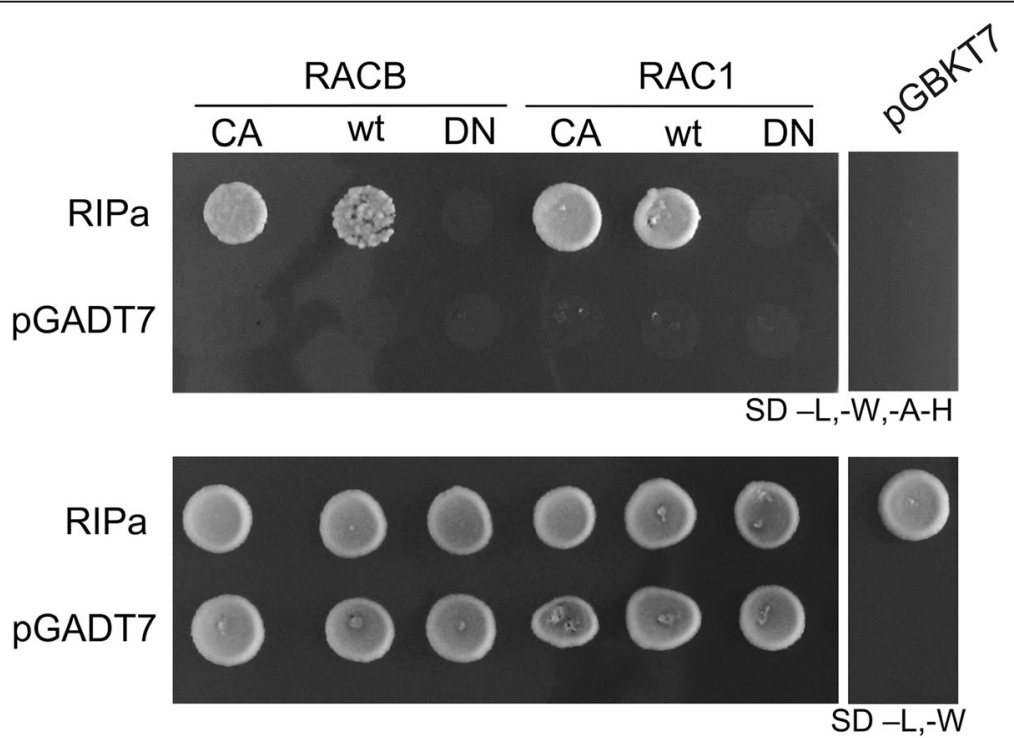

Fig. 1 Barley RIPa interacts with barley type I and type II ROPs in yeast. Bait- and prey-construct transformed yeast cells were dropped on either transformation-selected (SD -L-W) or interaction-selective (SD -L,-W,-A-H) medium. pGADT7 and pGBKT7 present empty vector controls to exclude auto-activity of respective ROP or RIPa constructs 


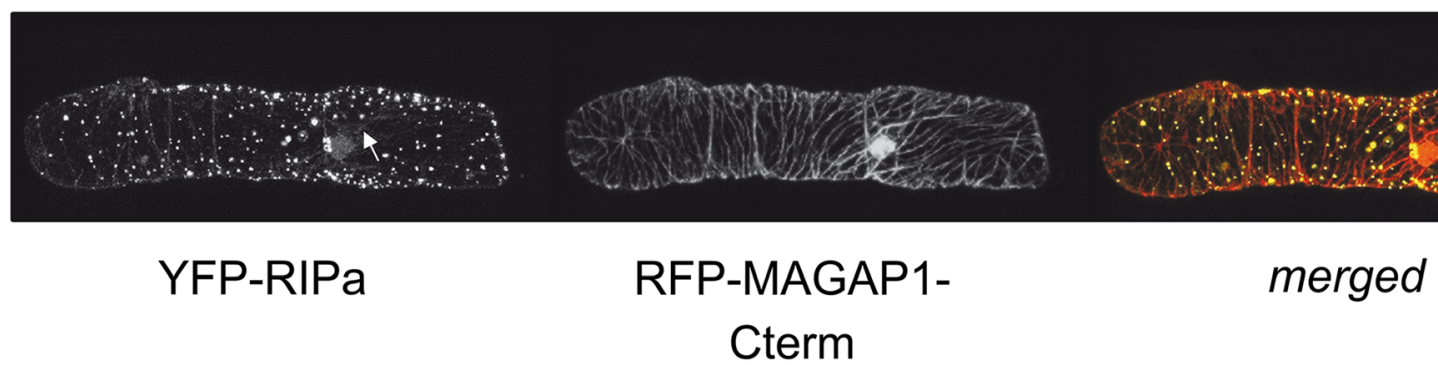

Fig. 2 Single cell-expressed barley YFP-RIPa localizes to immobile speckles and the cytoplasm (arrow in the YFP-RIPa channel). Whole cell Z-stack images were taken $24 \mathrm{~h}$ after biolistic transformation of barley epidermal cells. The MT-marker RFP-MAGAP1-Cterm was co-expressed to visualize MTs

localization of YFP-RIPa. RAC1 fully recruited YFP-RIPa to the cell periphery or plasma membrane and to a minor extent also to MTs, whereas CA RAC1 recruited YFPRIPa exclusively to the cell periphery/plasma membrane. DN RAC1 did not recuit YFP-RIPa or perhaps even enhanced protein aggregation in speckles (Fig. 3). Split YFP bimolecular fluorescence complementation (BiFC) experiments further confirmed that RAC1 and RIPa can interact in planta and that this interaction is taking place at the cell periphery and at MTs (Additional file 1, Figure S2). Together, data suggest that CA or wild type switchable RAC1 can influence the localization of YFP-RIPa most likely by direct protein interaction. In Fig. 3, a red fluorescing MT-marker was co-expressed. To further exclude that the marker influenced YFP-RIPa localization, we repeated the experiments with free mCherry as cytoplasmic and nucleoplasmic marker. Similar to what was observed before, CA RAC1 and also CA RACB recruited YFP-RIPa to the cell periphery, whereas DN RAC1 and DN RACB did not (Additional file 1, Figure S3).

\section{A ROP - ROP-GAP module positions RIPa in MT-restricted domains at the cell periphery}

Arabidopsis RIP3/MIDD1 localizes into MT-restricted membrane domains when co-expressed with the type II ROP ROP11, the catalytically active domain of ROPGEF4 and ROP-GAP3 [19]. We hence speculated that co-expression of the barley ROP-GAP MAGAP1 and the barley type II ROP RAC1 could modulate subcellular localization of YFP-RIPa. Therefore, we first confirmed that MAGAP1 can interact with RAC1 in yeast and in BiFC experiments and can recruit GFP-tagged MAGAP1 from MTs to the cell periphery/plasma membrane (Additional file 1, Figure S4). We had also found that MAGAP1 does not interact with RIPa in yeast (Additional file 1, Figure S1). We then used the MT marker DsRED-MAP 4 and co-expressed it with YFPRIPa, with untagged MAGAP1 and untagged RAC1. This led to accumulation of YFP-RIPa in MT-restricted domains at the cell periphery/plasma membrane. In this situation, MT-rich and YFP-RIPa-rich domains of the

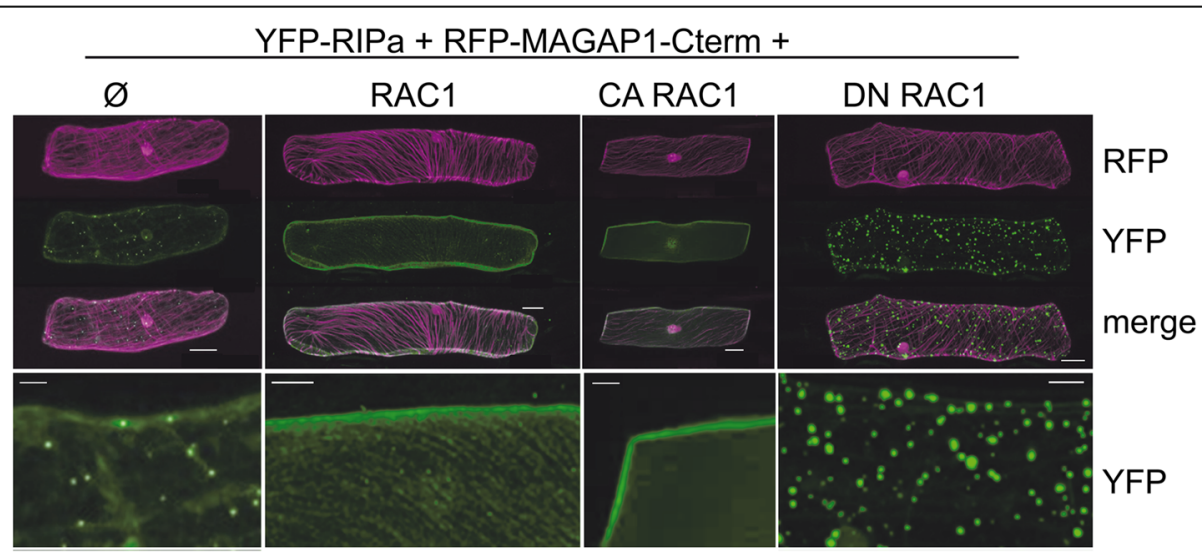

Fig. 3 Single cell expressed barley YFP-RIPa changes subcellular localization upon co-expression of untagged RAC1. YFP-RIPa alone ( $\varnothing$ ) localizes to immobile speckles and the cytoplasm. Co-expression of untagged RAC1 (WT RAC1) leads to plasma membrane and MT association of YFP-RIPS, co-expression of CA RAC1 leads to plasma membrane localization of YFP-RIPa and DN RAC1 leads to speckle-association of YFP-RIPa. The lower panels show digital magnifications of the YFP-RIPa signals with 40\% enhanced brightness. Whole cell Z-stack images were taken $24 \mathrm{~h}$ after biolistic transformation of barley epidermal cells. The MT-marker RFP-MAGAP1-Cterm was co-expressed to visualize MTs. Bars represent 20 um in the upper row and $5 \mu \mathrm{m}$ in the detail magnifications 


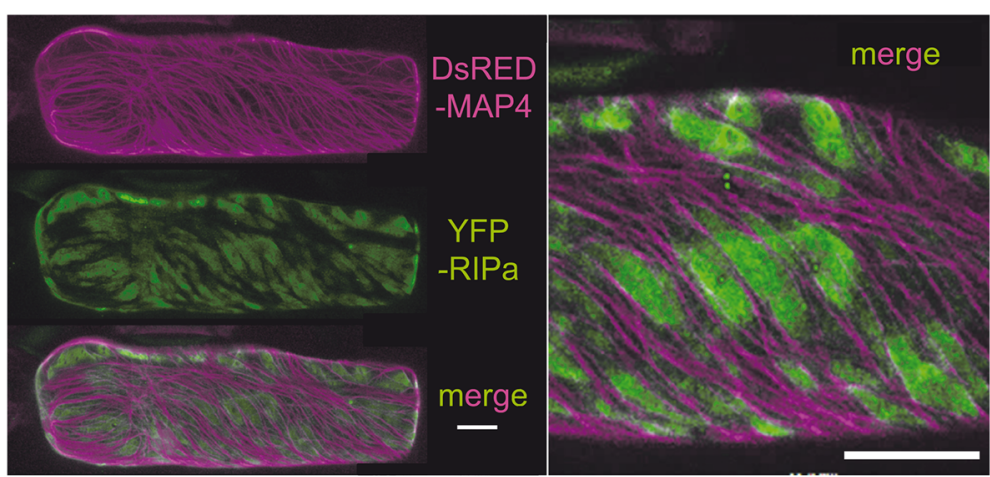

YFP-RIPa + MAGAP1 + RAC1 + DsRED-MAP4

Fig. 4 Barley YFP-RIPa localizes to MT-restricted membrane domains when co-expressed with wild type RAC1 and MAGAP1. Whole cell Z-stack images were taken $24 \mathrm{~h}$ after biolistic transformation of barley epidermal cells. The MT-marker DsRED-MAP 4 was co-expressed to visualize MTs. The right panel shows a digital magnification with 40\% enhanced brightness. Bars represent $20 \mu \mathrm{m}$

cell periphery mutually excluded each other (Fig. 4). Similar images were recorded when we used RFPMAGAP1-Cterm as an alternative MT marker (Fig. 5). Additionally, MTs appeared to function in formation or restriction of the YFP-RIPa-enriched domains because treatment with $30 \mu \mathrm{M}$ of the MT-depolymerizing drug oryzalin led to both disappearance of detectable MTs and the destruction of these domains and to more evenly peripheral localization of YFP-RIPa (Fig. 5). We also wanted to get more evidence for importance of MAGAP1 in heterogeneity of the YFP-RIPa distribution. Therefore, we co-expressed RAC1 and YFP-RIPa with different versions of labelled RFP-MAGAP1 to see whether a functional ROP-GAP is required to form the observed YFP-RIPa membrane domains. Next to full length RFP-MAGAP1 to observe membrane heterogeneity, we additionally used a MAGAP1 version, which lacked the carboxyterminal MT-assocciating domain (RFP-MAGAP1- $\Delta$ Cterm). This version is cytoplasmic when expressed alone but possesses functional ROPbinding CRIB and GAP domains. It is therefore recruited by activated ROPs to the cell periphery and fulfills a function in controlling ROP effects [9], As a third version, we used the MT marker RFP-MAGAP1-Cterm, which lacks the ROP-binding CRIB and GAP domains (see Fig. 6a for domain composition of MAGAP1 versions). In these experiments we did not co-express untagged MAGAP1. Again, co-expression of full length RFP-MAGAP1 resulted in patchy domains of YFP-RIPa at the cell periphery/plasma membrane, which were restricted by RFP-MAGAP1 labelled MTs. This depended on RAC1, because YFP-RIPa speckles occurred instead, when we omitted RAC1 (outer right panel in Fig. 6b). Interestingly, using RFP-MAGAP1-Cterm instead of full length RFP-MAGAP1, completely dissolved the accumulation of YFP-RIPa in specific membrane domains but showed YFP-RIPa distribution at the entire cell periphery/plasma membrane. Hence, the ROP-interacting domains of MAGAP1 appeared to be necessary for the formation of distinct YFP-RIPa-labelled membrane domains. Strikingly, when we used RFP-MAGAP1- $\triangle$ Cterm, this protein seemed to be recruited by RAC1 to the cell

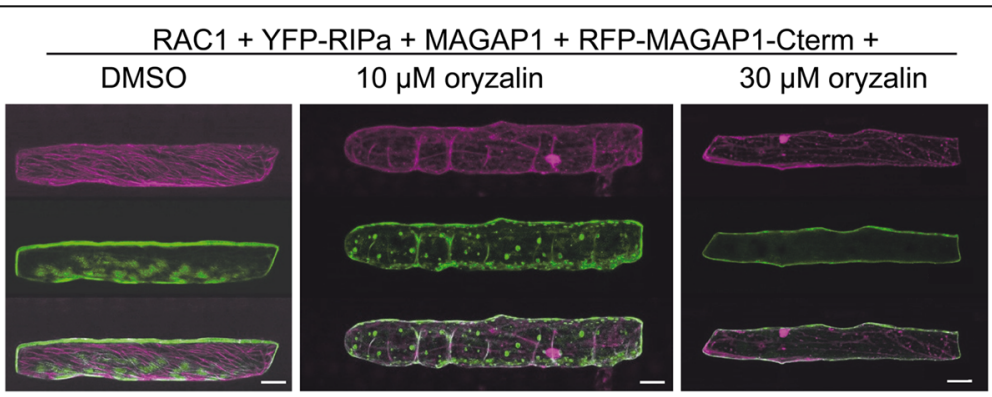

Fig. 5 Disturbance of YFP-RIPa localization to MT-restricted domains of the cell periphery/plasma membrane. YFP-RIPa (shown in green) when co-expressed with wild type RAC1 and MAGAP1 can be found in MT-restricted domains of the cell periphery/plasma membrane (see left panel for DMSO solvent control). This localization is dissolved when MTs are destroyed by either 10 or $30 \mu \mathrm{M}$ oryzalin treatment (solved in $0.25 \%$ [v/v] DMSO, treated for $3.2 \mathrm{~h}$ before imaging). Whole cell Z-stack images were taken $24 \mathrm{~h}$ after biolistic transformation of barley epidermal cells. The MT-marker RFP-MAGAP1-Cterm (shown in magenta) was co-expressed to visualize MTs. Bars represent $30 \mu \mathrm{m}$ 

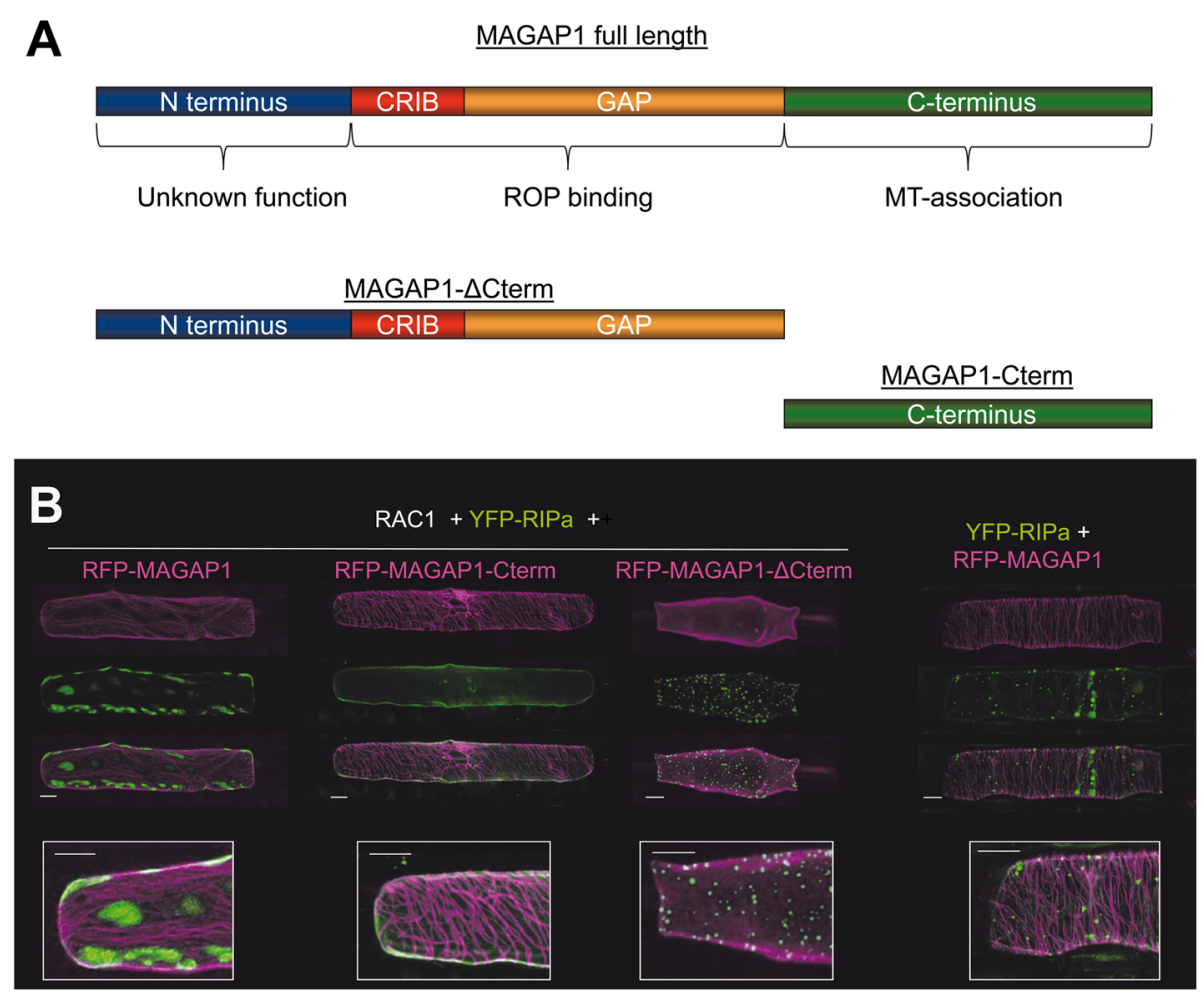

Fig. 6 Functional domains of MAGAP1 determine the formation of YFP-RIPa membrane domains. a Domain architecture of MAGAP1 and of truncated versions of MAGAP1, which were expressed as RFP fusion proteins. b Co-expression of fluorescent YFP-RIPa (shown in green) with untagged RAC1 (or without RAC1; outer right column) with three versions of RFP-MAGAP1 (shown in magenta) with or without ROP-binding and MT-association domains as depicted in $\mathbf{a}$. Whole cell Z-stack images were taken $24 \mathrm{~h}$ after biolistic transformation of barley epidermal cells. Bars represent $20 \mu \mathrm{m}$

periphery/plasma membrane and YFP-RIPa appeared again in speckles of unknown nature. This suggests that RFP-MAGAP1- $\triangle$ Cterm outcompeted YFP-RIPa from the interaction with RAC1 and hence a pattern occurred that is similar to that observed under co-expression of DN RAC1, which does not bind RIPa (compare Figs. 1 and 3). Together, this suggested that MAGAP1 can control asymmetric RAC1 activity, which is required for formation of RIPa-labelled membrane domains. To further test whether this strictly depends on GAP function of MAGAP1, we used MAGAP1-R185G. This mutant, lacks the catalytically active arginine residue, which mediates GTP hydrolysis for switching off RHO proteins. It can still bind ROPs but appears to be non-functional or even dominant negative as assessed by its functionality in limiting susceptibility to Bgh [9]. Interestingly, in presence of RFP-MAGAP1-R185G, YFP-RIPa enriched membrane domains could still form. However, cells also frequently showed diverse YFP-RIPa arrays without clear membrane associated heterogeneity. In these cells, we recorded YFP-RIPa at the entire cell periphery and MTs or in speckles (Additional file 1, Figure S5). This suggests, that MAGAP1 GAP activity is not strictly required for the formation of MT-restricted membrane domains with enriched RIPa but might contribute to the maintenance of the observed membrane patterns.

\section{RIPa accumulates at sites of fungal attack}

When transiently over-expressed in barley epidermal cells, CA RAC1 does not significantly support or inhibit penetration by $B g h$. We also did not measure a significant influence of transient RIPa over-expression on Bgh penetration success, when we applied the exact experimental protocol, in which RIPb over expression supports fungal penetration [22]. Yeast-two-hybrid assays did not suggest a direct interaction between RIPa and the Bgh virulence effector ROPIP1, which may target barley RACB but can also bind RAC1 in yeast [11] (Additional file 1, Figure S1). We hence wondered how YFP-RIPa would localize in interaction with $B g h$. When we inoculated leaves, in which we co-expressed YFP-RIPa, RAC1, MAGAP1 and the MT marker RFP-MAGAP1-Cterm, we detected, albeit somewhat less clear than in noninoculated leaves, patterns of mutually exclusive MTs and YFP-RIPa-labelled membrane domains. Additionally, YFP-RIPa clearly labelled a zone around the site of fungal attack likely representing plasma membrane that directly attached to the defensive cell wall apposition that 


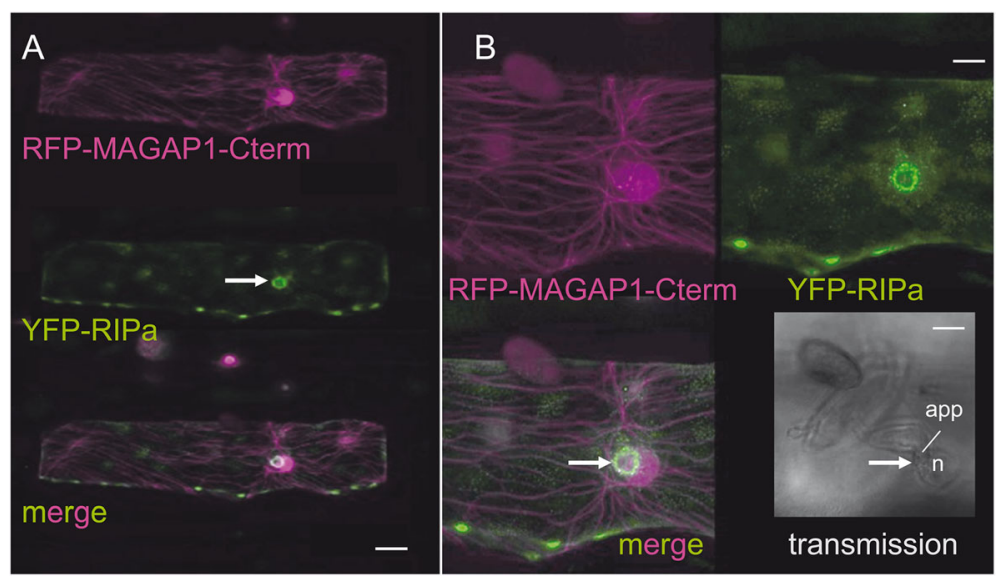

Fig. 7 YFP-RIPa localization at sites of fungal attack by Bgh. a Whole cell Z-stack images were taken $28 \mathrm{~h}$ after biolistic transformation of barley epidermal cells and $23 \mathrm{~h}$ after inoculation. The MT-marker RFP-MAGAP1-Cterm was co-expressed to visualize MTs. Bars represent $20 \mu \mathrm{m}$. Additionally, untagged RAC1 and untagged MAGAP1 are co-expressed. b Same cell as in A imaged at a higher zoom factor. Brightness was enhanced by $40 \%$ after imaging. Please note the fungal attack from an appressorium (app). YFP-RIPa is visible in membrane patches and around the site of attack (arrow). n, plant nucleus. Bars represent $8 \mu \mathrm{m}$

barley forms in response to the penetration attempt from the fungal appressorium (Fig. 7) [24]. Since we expressed RAC1 in its wild type form in these experiments, we also inoculated cells expressing YFP-RIPa under co-expression of CA RAC1 or DN RAC1. This revealed that YFP-RIPa localized to sites of fungal attack in cells with CA RAC1, too, but remained in unknown speckles, when co-expressed with DN RAC1 (Additional file 1, Figure S6).

\section{Discussion}

\section{$\mathrm{RIPa}$ is a ROP-binding protein}

Signaling RHO GTPases are crucial for cell polarity and cell development across the border of kingdoms. In plants, ROPs are increasingly well understood as molecular hubs that integrate signals from the cell periphery or apoplast and hormone responses to translate this into cellular organization of the cytoskeleton or membrane trafficking machinery. This serves among others polar cell development or response to pathogens and cell wall sensing [4, 25, 26]. To translate signaling cues into downstream-signaling ROP-GTP interacts with so-called ROP-effectors that either perform a direct function or serve as scaffolds for recruitment of other downstream factors, presumably in higher order complexes. The knowledge on plant ROP-effectors is constantly increasing but still very incomplete and for many ROP-effectors, we lack knowledge about the molecular mechanism, by which they control cellular organization [3]. Therefore and because ROP signaling is involved in plant resistance and susceptibility to diseases, we are interested in finding further ROP-effectors. We search for them in barley, because $i$. in monocot crops the knowledge on
ROP signaling is even less complete than in Arabidopsis, ii. barley ROPs are involved in pathogenesis of powdery mildew, and iii. The interaction of plants with powdery mildew fungi is a model system for studying the cell biology of plant-microbe interactions [27]. Based on what we and others found for ICR/RIP proteins in Arabidopsis, we identified the barley ICR/ RIP protein RIPa as a candidate ROP-effector. We found that it preferentially interacts with the activated form of both type I and type II ROPs. This is similar to ICR/RIPs of Arabidopsis, which interact with diverse ROPs in yeast. Additionally, there is also genetic interaction of ROPs and RIPs in planta [14, 15, 17, 18]. In addition to our yeast-based interaction assays, our BiFC results and the dynamics of subcellular RIPa localization upon co-expression of different versions of ROPs suggest that ROPs can interact with RIPa in planta. The fact that constitutively GTP-loaded CA RAC1 and wild type RAC1, which can be naturally loaded with GTP, strongly recruited RIPa to the cell periphery supports that RIPa interacts with signaling forms of ROPs such as RAC1-GTP at the plasma membrane. The partial accumulation of RIPa in unknown speckles, when overexpressed alone or with DN RAC1 or DN RACB further suggests that RIPa without a matching amount of binding partner forms aggregates or accumulates in unidentified cellular compartments. This is different to barley RIPb, which we recently found in the cytosol, at MTs and the cell periphery, when expressed alone. However, RIPb is naturally expressed on a higher level in the barley epidermis, when compared to RIPa, and hence might be also co-expressed with higher amounts of natural binding partners in the barley epidermis [22]. 
ROP activity and MTs control symmetry breaking of plasma membrane domains labelled by RIPa

The recuitment of RIPa by CA RAC1 or CA RACB suggested that the membrane association of RIPa depends on ROP signaling activity. We hence tested whether we can reconstitute a ROP-activation-deactivation module similar to what was reported for Arabidopsis xylem, in which RIP3/MIDD1 coordinates locally restricted cell wall apposition, and Nicotiana benthamiana epidermal cells. In these models, expression of ROP11, the catalytic domain of ROP-GEF4, ROP-GAP3 and RIP3/MIDD1 provokes symmetry breaking of the plasma membrane into zones with high and low ROP activity. This becomes visible by the presence of RIP3/MIDD1 in membrane domains of high ROP activity $[19,26]$. RIP3/ MIDD1 can further interact with kinesin13A in planta [17] and recruits this protein into areas of high ROP activity, where it supports kinesin13A-mediated depolymerization of MTs from the plus end. Vice versa, MTs laterally restrict RIP3/MIDD1-labelled ROP activity domains leading to lateral mutual inhibition of MTs and ROP activity and depletion of MTs from zones of high ROP11 activity [21]. Most recently, the same ROP11 that organizes MT patterns was shown to also organize filamentous actin at the side where secondary cell wall deposition is locally defined in xylem cells [28]. Interestingly, the expression of RAC1 and MAGAP1 together with RIPa appeared to be sufficient to reconstitute a MT-controlled ROP-activation-deactivation module in barley. Asymmetric appearance of RIPa at the plasma membrane in zones with very few or mostly lacking cortical MTs was reminiscent of the RIP3/MIDD1-labelled domains to ROP activity in Arabidopsis. We did not coexpress any ROP-GEF in these cells and hence it seems that the barley epidermis possesses sufficient endogenous GEF activity to activate RAC1. This is further supported because expression of wild type RAC1 similar to expression of CA RAC1 recruited RIPa to the plasma membrane in cells without co-expression of MAGAP1. We assume that RAC1 was activated by barley endogenous ROP-GEFs in these situations but hardly deactivated because no corresponding high amount of ROP-GAP was present in those cells, and ROPs have only a weak intrinsic GTP-hydrolyzing activity [29]. However, additional co-expression of either untagged MAGAP1 or RFP-tagged MAGAP1 led to symmetry breaking of the plasma membrane. MAGAP1 may not directly interact with RIPa but with activated RAC1 in these situations as our yeast-two-hybrid, BiFC and recruitment assays support. Hence, MAGAP1 might fulfil a complex function in forming and stabilizing membrane heterogeneity. On the one hand, MAGAP1 is a classical ROP-GAP with a CRIB domain that supports binding to ROP-GTP and possesses a conserved catalytical arginine residue, which is predicted to hydrolyze ROP-bound GTP and appears to be required for the control of ROP effects [9]. On the other hand, MAGAP1 is directly associated to MTs by its carboxyterminal domain and hence ideally positioned to perform a function in spatial feedback from MTs. This is different from Arabidopsis ROP-GAP3 for which no MT-association is reported. The idea, that MAGAP1 indeed functions in lateral restriction of ROP activity domains in barley is strongly supported by the expression of truncated versions of MAGAP1, which interfered with membrane symmetry breaking. RIPa speckles were observed, when we co-expressed RAC1 with MAGAP1- $\Delta$ Cterm, which is detached from MTs by truncation of its $\mathrm{C}$-terminus but possesses intact domains for ROP-GTP interaction and GTP hydrolysis [9]. Functionality in restricting ROP activity is supported for MAGAP1$\Delta$ Cterm because it is fully functional in limiting susceptibility to $B g h$ [9]. In this situation, MAGAP1- $\Delta$ Cterm occurred at the plasma membrane, to which it was most likely recruited by the co-expressed RAC1. It was similarly shown before that co-expressed $\mathrm{CA}$ RACB can recruit soluble MAGAP1- $\Delta$ Cterm to the cell periphery [9]. We therefore speculate that MAGAP1- $\Delta$ Cterm outcompetes RIPa from binding to RAC1 in this situation and additionally functions as a ROP-GAP such that most of the expressed RAC1 is deactivated immediately after loading GTP. Together, this could explain occurrence of RIPa in speckles, in which it otherwise was observed without co-expression of RAC1 or upon co-expression of DN RAC1. Hence, RIPa and MAGAP1 may rather compete for binding than forming a ternary protein complex with RAC1.

RAC1-dependent membrane domains of RIPa formed under co-expression of MAGAP1. By contrast, RIPa more symmetrically labelled the cell periphery when MAGAP1-Cterm was co-expressed, which does not possess any ROP binding or GAP domain but still localizes to MTs. This also shows that MTs did not serve as a pure physical barrier to the diffusion of RIPa or RAC1 activity but as a physiological barrier dependent on the presence of full length MAGAP1. In this context, it is interesting that RIPa-labeled membrane domains did never form without MAGAP1. This suggests that MAGAP1 does not only control ROP activity but possesses also characteristics of a ROP effector, which is involved in ROP activity-dependent cell polarity. Enzymatically inactive MAGAP1-R185G was partially functional in allowing the formation of membrane heterogeneity. This mutant, however, caused little consistency in the patterns of YFP-RIPa localization suggesting that hydrolytic GAP activity is likely involved in stabilizing asymmetric membrane patterns. This would be best explained if MAGAP1 is recruited by activated RAC1 for the support of GTP hydrolysis in the vicinity of MTs where MAGAP1 is sequestered. MAGAP1 had been similarly suggested before to function in MT-associated negative feedback on ROP activity in barley pathogen defense [9, 30]. However, it remains unclear how 
MAGAP1 supports initiation of RIPa-labeled patterning of the plasma membrane. We further observed MT-depletion from RIPa-labeled membrane domains but so far lack evidence that barley kinesin 13A-like proteins might be involved in this process as shown for MT-depletion in Arabidopsis [21]. Together, functions of MAGAP1 and the spatial control of these functions near MTs appear necessary for symmetry breaking of ROP activity at the plasma membrane (see Fig. 8 for a model).

\section{RIPa might label a membrane domain of high ROP activity in interaction with Bgh}

In Bgh-attacked cells, RIPa was also observed in membrane domains, when co-expressed with RAC1 and MAGAP1. However, the lateral restriction of RIPadomains by MTs was less distinct. The overall intensity of RIPa labelling of the membrane was not very high when contrasted by local accumulation at the site of fungal infection. Because RIPa seems to preferentially accumulate at sites of high ROP or more specifically RAC1 activity, this might indicate that RAC1 can be activated at sites of fungal attack. This is reminiscent of the accumulation of further ROP activity sensors such as RIC171 or RIPb at sites of fungal attack [22, 31]. Together, these observations support earlier hypotheses of locally enhanced ROP activity at sites where Bgh attempts to penetrate $[30,31]$.

The physiological effect of this local ROP activity is not well understood and RIPa has no significant effect on the fungal penetration success when over-expressed [22]. RAC1 seems to be involved in modulation of fungal penetration success in barley but this depends on whether CA RAC1 was expressed transiently or stably and on whether Bgh or Magnaporthe oryzae was attacking $[7,12]$. The putative rice ortholog of barley RAC1 is also called RAC1. Rice RAC1 functions in chitintriggered immunity and is activated via the chitin-

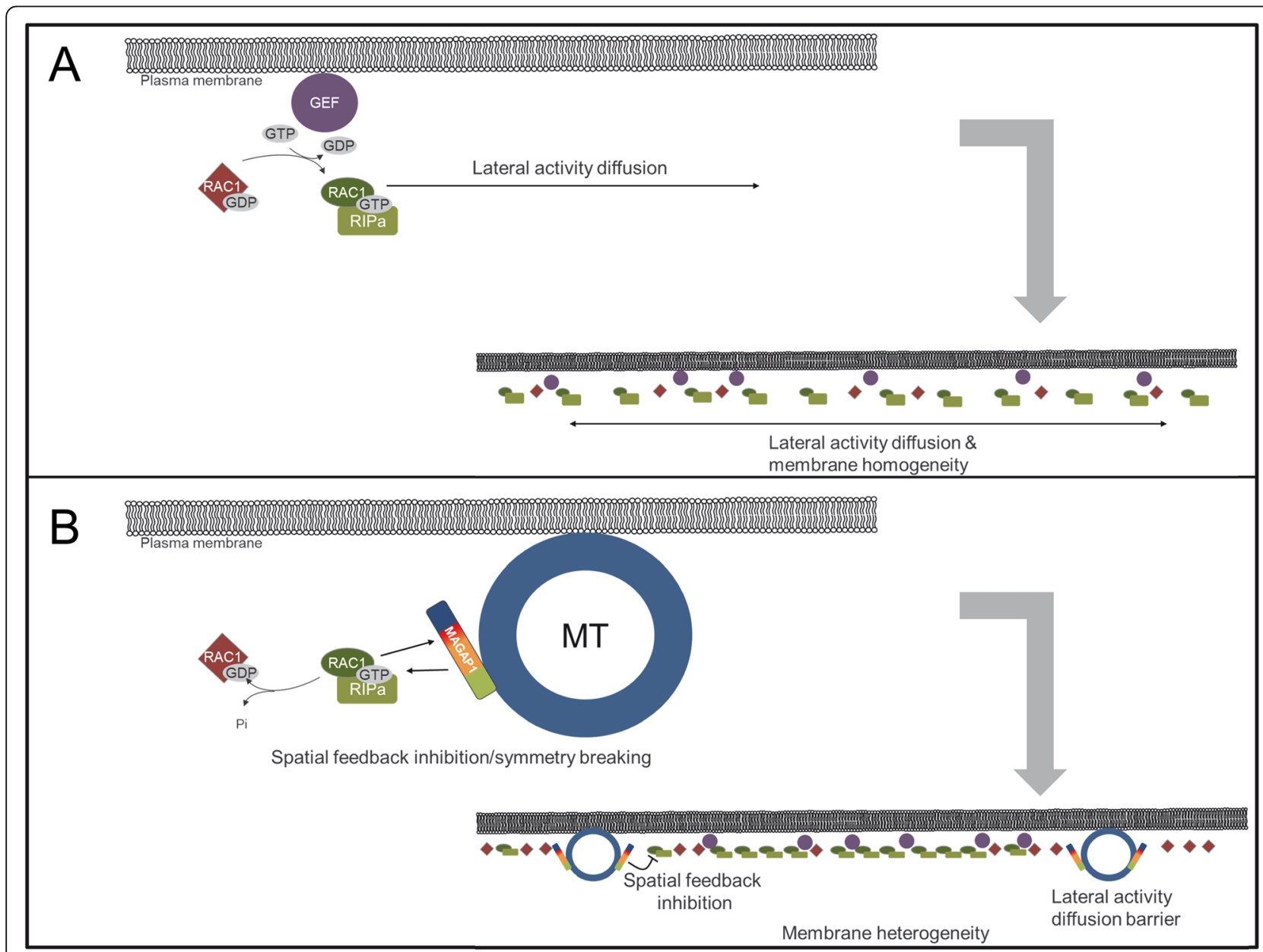

Fig. 8 MT and MAGAP1-dependent symmetry breaking of plasma membrane-associated RAC1-RIPa signaling. a In absence of MTs or MAGAP1, GEF-supported RAC1 activity can freely diffuse at the plasma membrane and RIPa is evenly distributed. $\mathbf{b}$ In presence of intact MTs and functional MT-associated MAGAP1, MAGAP1 laterally inhibits RAC1 activity from MTs. This leads to spatially restricted negative feedback, and hence symmetry breaking and membrane heterogeneity 
signaling receptor kinase CERK1 and RAC-GEF1, a member of a plant-specific RHO-GEF family [32]. Chitin is a potent elicitor of early defense reactions in barley and can induce systemic resistance to Bgh infection [10, 33]. However, it is unclear to what extent chitin elicitation contributes to basal resistance of barley in the authentic interaction with Bgh. We thus can only speculate that chitin elicitation is also involved in local activation of RAC1 in barley but this would explain why we observe local enrichment of the RAC1 activity sensor RIPa at sites where we can assume chitin elicitors from the fungal cell wall to be present.

\section{Conclusions}

Data suggest that barley RIPa interacts with barley ROPs and specifies RAC1-activity associated membrane domains with potential signaling capacity. Lateral diffusion of this RAC1 signaling capacity is restricted by microtubules and MICROTUBULE-ASSOCIATED ROP-GTPASE ACTIVATING PROTEIN 1. Hence, an interplay of ROP activity and spatially confined MT-associated enzymatic restriction of ROP activity by MAGAP1 can provoke and stabilize asymmetry at the plasma membrane of barley epidermal cells. Resulting membrane heterogeneity potentially reflects a mechanism by which monocot cells focus ROP activity comparable to what was reported before for dicots. Focal accumulation of RIPa at sites of fungal attack may further indicate locally restricted ROP activity at sites of fungal invasion.

\section{Methods}

\section{Plant and fungal material}

We used the barley (Hordeum vulgare) cultivar Golden Promise for transformation and inoculation experiments. The material was obtained from the Leibniz Institute of Plant Genetics and Crop Plant Research in Gatersleben, Germany. We grew plants with a light dark cycle of 16 $\mathrm{h} / 8 \mathrm{~h}$ at light intensity of $150 \mu \mathrm{M} \mathrm{s}^{-1} \mathrm{~m}^{-2}$ and $65 \%$ relative humidity and at $18{ }^{\circ} \mathrm{C}$. Blumeria graminis f.sp. hordei race A6 was maintained on Golden Promise plants under the same conditions inoculated on plants by shaking plants with sporulating powdery mildew and blowing spores into a plastic tower $(200 \times 50 \times 50 \mathrm{~cm})$, which we had positioned over the naïve plants or transformed leaf segments on agar plates.

\section{Construction of expression constructs}

Barley RIPa (HORVU3Hr1G087430) was amplified from cDNA using gene-specific start to stop primers equipped with Xba1_fwd and Xba1_rev restriction sites for subcloning (RIPaXbaI_fw 5'-TCTAGATATGCAGACAGC CAAGACAAG-3'; RIPaXbaI_rv 5' - TCTAGATCAT TTCTTCCACATTCCACTG-3'). We ligated the amplicons into the pGEM-T easy vector (Promega, Madison, WI, USA) by blunt end cloning according to the manufacturer's instructions and sequenced the inserts. For Yeast Two-Hybrid assays $R I P a$ was subcloned from the pGEM-T easy vector into pGADT7 plasmid (Clontech Laboratories) using the mentioned restriction sites. For over-expression and protein localization we used the high copy pGY1 plasmid, containing the CaMV35S promotor. We cut the RIPa insert by Xba1 from the pGEM$\mathrm{T}$ easy vector and ligated $H v R I P a$ into the pGY1 plasmid or pGY1-YFP (without YFP STOP codon) plasmid to gain a N-terminal YFP fusion construct pGY1-YFP-RIPa. Orientation was confirmed by sequencing. For cloning into the Y2H pGADT7 vector, RIPa was amplified with RIPa_Nde 5'- TGGATCCTCATTTCTTCCACAT TCCACTG-3' and RIPa_BamH1 5'-ACATATGCAG ACAGCCAAGACAAGG-3'. Construction of plant expression and $\mathrm{Y} 2 \mathrm{H}$ vectors for barley MAGAP1, RAC1 and RACB variants was described previously [7, 9, 31]. Also, the construction of MAGAP1, RFP-MAGAP1 and truncated versions of this was described previously [9]. For BiFC, RAC1, MAGAP1 or RIPa were amplified from the pGEM-T easy vectors using gene specific primers with attached restriction enzyme cleavage sites. The PCR products were then digested into cloning sites of pUC-SPYNE(R)173 or pUC-SPYCE (MR) plasmids [34] using these restriction sites.

\section{Biolistic transformation of barley leaf segments}

We transformed barley epidermal cells by biolistic particle bombardment with PDS-1000/HE (Biorad, Hercules, CA; USA) as described earlier [35]. Therefore, we placed segments of $7 \mathrm{~d}$ old primary leaves of barley on $0.8-1 \%(\mathrm{w} / \mathrm{v})$ water-agar. For each shot, we precipitated $1 \mu \mathrm{g}$ plasmid DNA on $302.5 \mu \mathrm{g}$ of $1 \mu \mathrm{m}$ gold particles (Biorad, Hercules, CA, USA) by adding the same volume of $1 \mathrm{M} \mathrm{CaCl}_{2}$. Half the DNA amount was used for pGY1-mCherry transformation markers. Finally, we added $3 \mu \mathrm{l}$ per shot of $2 \mathrm{mg} / \mathrm{ml}$ protamine (Sigma). We subsequently ( $30 \mathrm{~min}$ later at RT) washed twice the plasmid-coated gold with $500 \mu \mathrm{l}$ of first $70 \%(\mathrm{v} / \mathrm{v})$ and second $100 \%$ ethanol. The re-suspendend gold particle were then pipetted $(6 \mu \mathrm{l})$ on the macro carrier for bombardment.

\section{Subcellular localization and protein recruitment in planta} Localization of YFP-HvRIPa either expressed alone or simultaneously with different versions of RAC1, RACB and MAGAP1 was performed at the indicated time points after transient transformation of barley leaves. We imaged single transformed cells with a Leica TCS SP5 confocal laser scanning microscope and the use of hybrid $\mathrm{HyD}$ detectors. Excitation and emission wavelength were individually adapted to the respective fluorophores as described before and imaged were recorded 
by sequentially scanning line-by-line with a 3-times averaging $[9,31]$.

\section{Yeast two-hybrid assays}

Constructs were transformed into yeast strain AH109 following the small-scale LiAc yeast transformation procedure from the Yeast Protocol Handbook (Clontech, Mountain View, CA, USA). Bait- and prey-construct transformed yeast cells were dropped on either transformation-selected (SD -L-W) or interaction-selective (SD - L,-W,-A-H) medium. pGADT7 and pGBKT7 were included as empty vector controls to exclude auto-activity of respective constructs.

\section{Supplementary information}

Supplementary information accompanies this paper at https://doi.org/10 1186/s12870-020-2299-4

Additional file 1: Supplementary Figure S1. Barley RIPa interacts with itself in yeast. Bait- and prey-construct transformed yeast cells were dropped on either transformation-selected (SD -L-W) or interactionselective (SD - $\mathrm{L},-\mathrm{W},-\mathrm{A}-\mathrm{H}$ ) medium. pGADT7 presents empty vector controls to exclude auto-activity of respective constructs. Supplementary Figure S2. Bimolecular fluorescence complementation of split YFP suggests direct interaction of RIPa and RAC1 in planta. A. In planta interaction of YFP ${ }_{N}-R I P a$ and $Y_{P F}-R A C 1$ allows for YFP fluorescence complementation at the cell periphery and at MTs (column one). Fluorescence is faint when one of the split YFP proteins is expressed alone instead of being fused to RIPa or RAC1 (columns three and four). Fusion of YFP $_{C}$ to the dominant negative mutant RAC1-T28N does not interact with $\mathrm{RIPa}$, and no fluorescence is observed (column two). Red fluorescing dsRED protein is localized in the cytoplasm and nucleoplasm and contrasts YFP fluorescence at the cell periphery. The pictures show projections of 20-30 optical sections through the epidermal cell at $2 \mu \mathrm{m}$ increments. All signals have been recorded with the same microscope settings. B. Enlargement of the $\mathrm{YFP}_{\mathrm{N}}-\mathrm{RIPa}-\mathrm{YFP} \mathrm{P}_{\mathrm{C}}-\mathrm{RAC1}$ split YFP signal from column 1 in A. Brightness of the picture was enhanced by $40 \%$. Supplementary Figure S3. Barley YFP-RIPa localizes to the cell periphery when co-expressed with CA RAC1 or CA RACB (left panels) and to speckles of unknown nature when co-expressed with DN RAC1 or DN RACB (right panels. Whole cell Z-stack images were taken $24 \mathrm{~h}$ after biolistic transformation of barley epidermal cells. The cytosolic marker mCherry was co-expressed to contrast the cytoplasm. Supplementary Figure S4. Interaction between MAGAP1 and RAC1. A. Barley MAGAP1 interacts with the barley type II ROP RAC1 in yeast. Bait- and prey constructtransformed yeast cells were dropped on either transformation-selected (SD -L-W) or interaction-selective (SD - L,-W,-A-H) medium. PGADT7 and pGBKT7 represent an empty vector control to exclude auto-activity of the MAGAP1 construct. B. Bimolecular fluorescence complementation of split YFP suggests direct interaction of MAGAP1 and RAC1-GTP in planta. In planta interaction of YFP $\mathrm{N}_{\mathrm{N}}-\mathrm{MAGAP1}$ and $\mathrm{YPF}_{\mathrm{C}}-\mathrm{CARAC1}$ allows for YFP fluorescence complementation at the cell periphery (column one). Fluorescence is faint when YPFC-DNRAC1 is co-expressed instead activated RAC1 (columns two). In this case, background fluorescence complementation is similar to what is observed when free YFP $_{N}$ instead of YFP $\mathrm{N}^{-}$ MAGAP1 is coexpressed (column three). The pictures show projections of 20-30 optical sections through the epidermal cell at $2 \mu \mathrm{m}$ increments. All signals have been recorded with the same microscope settings. $C$. Change of GFPMAGAP1 localization upon co-expression of CA RAC1. Whole cell Z-stack images were taken $24 \mathrm{~h}$ after biolistic transformation of barley epidermal cells. GFP-MAGAP1 localizes to MTs but is recruited to the cell periphery upon co-expression of CA RAC1. EV, empty vector. Lower panel: The cytosolic marker DsRED was co-expressed. Bars represent $30 \mu \mathrm{m}$. Supplementary Figure S5. Co-expression of RAC1 and the presumably GAP-inactive mutant MAGAP1R185G leads to inconsistent RIPa localization. Co-expression of fluorescent YFP-RIPa with untagged RAC1 and with RFPMAGAP1R185G. Whole cell Z-stack images of three representative cells with diverse locasiation patterns of YFP-RIPa were taken $24 \mathrm{~h}$ after biolistic transformation of barley epidermal cells. Bars represent $20 \mu \mathrm{m}$. Supplementary Figure S6. YFP-RIPa localization at sites of fungal attack by Bgh but not when DN RAC1 is co-expressed. Whole cell Z-stack images were taken $28 \mathrm{~h}$ after biolistic transformation of barley epidermal cells and $23 \mathrm{~h}$ after inoculation. Additionally, untagged CA RAC1 or DN RAC1 are co-expressed. Brightness was enhanced by $20 \%$ after imaging. Please note the fungal attack from an appressorium (app). Site of attack, arrow; hau; fungal haustorium. Long arrows mark plasma membrane folds at cell wall protrusions at the cell bottom facing mesophyll cells. Bars represent $20 \mu \mathrm{m}$.

\section{Abbreviations}

Bgh: Blumeria graminis f.sp. hordei; CA: Constitutively activated; CRIB: CDC42/ RAC-Interactive Binding; DN: Dominant negative; GAP: GTPase-activating protein; GEF: Guanine nucleotide exchange factors; ICR: Interactor of Constitutive Active ROPs; MAGAP1: MICROTUBULE-ASSOCIATED ROP-GTPASE ACTIVATING PROTEIN 1; MIDD1: Microtubule depletion domain 1; MT: Microtubule; RAC: Ras (Rat sarcoma)-related C3 botulinum toxin substrate 1; RIC: ROP-Interactive CRIB motif-containing proteins; RIP: ROPInteractive Partner; ROP: RHO of plants

\section{Acknowledgements}

We are grateful to Stefan Engelhardt (TU Munich, Chair of Phytopathology) for technical advice and fruitful discussions and to Vera Schnepf (TU Munich Phytopathology) who performed pilot experiments on barley RIPa.

\section{Authors' contributions}

$\mathrm{RH}$ developed the research questions, designed the study, prepared figures and wrote the manuscript. $\mathrm{CH}$ and $\mathrm{CM}$ designed and performed the experiments and prepared figures. All authors have read and approved the manuscript.

\section{Funding}

The project was funded in frame of research grants from the German Research Foundation to RH (DFG HU886-8). The funders had no role in the experiment design, data analysis, decision to publish, or preparation of the manuscript.

\section{Availability of data and materials}

Data sharing is not applicable to this article as no quantitative datasets were generated or analysed during the current study. Original microscopic picture files, constructs and seeds are available upon request from TUM.

Ethics approval and consent to participate

Not applicable.

\section{Consent for publication}

Not applicable.

\section{Competing interests}

The authors declare that they have no competing interests.

Received: 4 September 2019 Accepted: 21 February 2020

Published online: 02 March 2020

\section{References}

1. Lawson CD, Ridley AJ. Rho GTPase signaling complexes in cell migration and invasion. J Cell Biol. 2018:217:447-57.

2. Zheng ZL, Yang ZB. The Rop GTPase: an emerging signaling switch in plants. Plant Mol Biol. 2000;44:1-9.

3. Feiguelman G, Fu Y, Yalovsky S. ROP GTPases structure-function and signaling pathways. Plant Physiol. 2018;176:57-79.

4. Kawano Y, Kaneko-Kawano T, Shimamoto K. Rho family GTPase-dependent immunity in plants and animals. Front Plant Sci. 2014;5:522.

5. Kost B. Spatial control of Rho (Rac-Rop) signaling in tip-growing plant cells. Trends Cell Biol. 2008;18:119-27.

6. Platre MP, Bayle V, Armengot L, Bareille J, Marques-Bueno MDM, Creff A Maneta-Peyret L, Fiche JB, Nollmann M, Miege C, et al. Developmental control of plant Rho GTPase nano-organization by the lipid phosphatidylserine. Science. 2019;364:57-62. 
7. Schultheiss H, Dechert C, Kogel KH, Hückelhoven R. Functional analysis of barley RAC/ROP G-protein family members in susceptibility to the powdery mildew fungus. Plant J. 2003;36:589-601.

8. Schultheiss $\mathrm{H}$, Dechert $\mathrm{C}$, Kogel $\mathrm{KH}$, Hückelhoven R. A small GTP-binding host protein is required for entry of powdery mildew fungus into epidermal cells of barley. Plant Physiol. 2002;128:1447-54.

9. Hoefle C, Huesmann C, Schultheiss H, Boernke F, Hensel G, Kumlehn J, Hückelhoven R. A barley ROP GTPase ACTIVATING PROTEIN associates with microtubules and regulates entry of the barley powdery mildew fungus into leaf epidermal cells. Plant Cell. 2011:23:2422-39.

10. Scheler B, Schnepf V, Galgenmuller C, Ranf S, Huckelhoven R. Barley disease susceptibility factor RACB acts in epidermal cell polarity and positioning of the nucleus. J Exp Bot. 2016;67:3263-75.

11. Nottensteiner M, Zechmann B, McCollum C, Huckelhoven R. A barley powdery mildew fungus non-autonomous retrotransposon encodes a peptide that supports penetration success on barley. J Exp Bot. 2018;69:3745-58.

12. Pathuri IP, Zellerhoff N, Schaffrath U, Hensel G, Kumlehn J, Kogel K-H, Eichmann R, Hückelhoven R. Constitutively activated barley ROPs modulate epidermal cell size, defense reactions and interactions with fungal leaf pathogens. Plant Cell Rep. 2008;27:1877-87.

13. Huesmann C, Reiner T, Hoefle C, Preuss J, Jurca ME, Domoki M, Fehér A, Hückelhoven R. Barley ROP binding kinase1 is involved in microtubule organization and in basal penetration resistance to the barley powdery mildew fungus. Plant Physiol. 2012;159:311-20.

14. Lavy M, Bloch D, Hazak O, Gutman I, Poraty L, Sorek N, Sternberg H, Yalovsky S. A novel ROP/RAC effector links cell polarity, root-meristern maintenance, and vesicle trafficking. Curr Biol. 2007;17:947-52.

15. Li S, Gu Y, Yan A, Lord E, Yang ZB. RIP1 (ROP Interactive Partner 1)/ICR1 marks pollen germination sites and may act in the ROP1 pathway in the control of polarized pollen growth. Mol Plant. 2008;1:1021-35.

16. Wu G, Gu Y, Li SD, Yang ZB. A genome-wide analysis of Arabidopsis Ropinteractive CRIB motif-containing proteins that act as Rop GTPase targets. Plant Cell. 2001;13:2841-56.

17. Mucha E, Hoefle C, Hückelhoven R, Berken A. RIP3 and AtKinesin-13A-A novel interaction linking rho proteins of plants to microtubules. Eur J Cell Biol. 2010;89:906-16

18. Oda $Y$, lida $Y$, Kondo $Y$, Fukuda H. Wood cell-wall structure requires local 2D-microtubule disassembly by a novel plasma membrane-anchored protein. Curr Biol. 2010;20:1197-202.

19. Oda Y, Fukuda H. Initiation of cell wall pattern by a rho- and microtubuledriven symmetry breaking. Science. 2012;337:1333-6.

20. Hazak O, Bloch D, Poraty L, Sternberg H, Zhang J, Friml J, Yalovsky S, Leyser O. A Rho scaffold integrates the secretory system with feedback mechanisms in regulation of Auxin distribution. PLoS Biol. 2010;8: e1000282.

21. Oda Y, Fukuda H. Rho of plant GTPase signaling regulates the behavior of Arabidopsis Kinesin-13A to establish secondary cell wall patterns. Plant Cell. 2013;25:4439-50.

22. McCollum C, Engelhardt S, Hückelhoven R. ROP INTERACTIVE PARTNER b interacts with the ROP GTPase RACB and supports fungal penetration into barley epidermal cells. bioRxiv. 2019:750265. https://doi.org/10.1101/750265.

23. Lavy M, Yalovsky S. Association of Arabidopsis type-II ROPs with the plasma membrane requires a conserved C-terminal sequence motif and a proximal polybasic domain. Plant J. 2006;46:934-47.

24. Zeyen RJ, Carver TLW, Lyngkjaer MF. Epidermal cell papillae. Powdery mildews: a comprehensive treatise; 2002. p. 107-25.

25. Wolf S, Hematy K, Hofte H. Growth control and cell wall signaling in plants. Annu Rev Plant Biol. 2012;63:381-407.

26. Yang Z, Lavagi I. Spatial control of plasma membrane domains: ROP GTPase-based symmetry breaking. Curr Opin Plant Biol. 2012;15:601-7.

27. Hückelhoven R, Panstruga R. Cell biology of the plant-powdery mildew interaction. Curr Opin Plant Biol. 2011;14:738-46.

28. Sugiyama Y, Nagashima Y, Wakazaki M, Sato M, Toyooka K, Fukuda H, Oda Y. A Rho-actin signaling pathway shapes cell wall boundaries in Arabidopsis xylem vessels. Nat Commun. 2019;10:468.

29. Berken A. ROPs in the spotlight of plant signal transduction. Cell Mol Life Sci. 2006;63:2446-59.

30. Dörmann P, Kim H, Ott T, Schulze-Lefert P, Trujillo M, Wewer $V$, Hückelhoven R. Cell-autonomous defense, re-organization and trafficking of membranes in plant-microbe interactions. New Phytol. 2014;204:815-22
31. Schultheiss H, Preuss J, Pircher T, Eichmann R, Hückelhoven R. Barley RIC171 interacts with RACB in planta and supports entry of the powdery mildew fungus. Cell Microbiol. 2008;10:1815-26.

32. Akamatsu A, Wong HL, Fujiwara M, Okuda J, Nishide K, Uno K, Imai K, Umemura K, Kawasaki T, Kawano Y, Shimamoto K. An OsCEBiP/OsCERK1OsRacGEF1-OsRac1 module is an essential early component of chitininduced rice immunity. Cell Host Microbe. 2013;13:465-76.

33. Kasbauer CL, Pathuri IP, Hensel G, Kumlehn J, Huckelhoven R, Proels RK. Barley ADH-1 modulates susceptibility to Bgh and is involved in chitininduced systemic resistance. Plant Physiol Biochem. 2018;123:281-7.

34. Waadt R, Schmidt LK, Lohse M, Hashimoto K, Bock R, Kudla J. Multicolor bimolecular fluorescence complementation reveals simultaneous formation of alternative CBL/CIPK complexes in planta. Plant J. 2008;56:505-16.

35. Douchkov D, Nowara D, Zierold U, Schweizer P. A high-throughput genesilencing system for the functional assessment of defense-related genes in barley epidermal cells. Mol Plant-Microbe Interact. 2005;18:755-61.

\section{Publisher's Note}

Springer Nature remains neutral with regard to jurisdictional claims in published maps and institutional affiliations.

\section{Ready to submit your research? Choose BMC and benefit from:}

- fast, convenient online submission

- thorough peer review by experienced researchers in your field

- rapid publication on acceptance

- support for research data, including large and complex data types

- gold Open Access which fosters wider collaboration and increased citations

- maximum visibility for your research: over $100 \mathrm{M}$ website views per year

At BMC, research is always in progress.

Learn more biomedcentral.com/submissions 